\title{
Immunohistochimie de la peau humaine irradiée
}

\author{
B. BENYAHIA, H. MAGDELENAT \\ Institut Curie, 26, rue d'Ulm, 7500 Paris
}

L'irradiation thérapeutique des cancers peut donner lieu à des lésions tantôt précoces, de nature inflammatoire, tantôt tardives, non résolutives spontanément, liées à une sclérose d'installation progressive, tant dermique que stromale. Quarante prélèvements de peau mammaire humaine irradiée ont été étudiés comparativement avec des fragments cutanés non irradiés, par immunohistochimie, à la recherche de modifications du phénotype des fibroblastes dermiques, défini par les caractères du cytosquelette, de la répartition de la fibronectine tissulaire, de l'expression de facteurs de croissance (transforming growth factor BTGF B) ou de récepteurs de ces facteurs de croissance (récepteurs de l'epidermal growth factor -EGFR- et du platelet derived growth factor PDGFR- $\alpha$ ou $\beta)$.

Les fibroblastes dermiques de peau irradiée comportent toujours des filaments intermédiaires de vimentine (V) et, pour nombre d'entre eux, des microfilaments d' $\alpha$-actine de muscle lisse (A) ; les filaments de desmine (D) étaient constamment absents. Ce phénomène $V+A+D$ - permet de les identifier à des « myofibroblastes » ou fibroblastes réactionnels. Une accumulation de la fibronectine est observée en réseau au niveau du tissu conjonctif du derme cutané, le long de la membrane des fibroblastes mais également entre les cellules basales et parabasales épidermiques. La localisation membranaire de l'EGFR est visualisée au niveau de l'ensemble des couches cellulaires de l'épiderme et ce caractère représente un signe constant d'irradiation antérieure. Sur les coupes de peau irradiée, le TGFß est mis en évidence dans les noyaux des fibroblastes dermiques et des cellules parabasales et intermédiaires de l'épiderme. Enfin, si la présence de PDGFR $\alpha$ est inconstante, des récepteurs de PDGFß sont identifiés sur la membrane cellulaire des fibroblastes dermiques de peau irradiée, alors qu'ils ne sont jamais visualisés dans le derme de peau non irradiée.

L'ensemble de ces données laisse supposer l'existence d'interactions complexes entre les fibroblastes du derme irradié et, d'une part, les cellules épidermiques, d'autre part les constituants de la matrice extracellulaire ; tout ou partie de ces interactions sont très certainement médiées par des facteurs, tels que le TGFB, l'EGF et le PDGFB. 